\title{
Simulation of Apparent Infection Rate to Predict Severity of Soybean Rust Using a Fuzzy Logic System
}

\author{
K. S. Kim, T. C. Wang, and X. B. Yang \\ First and third authors: Department of Plant Pathology, Iowa State University, Ames 50011; and second author: Asian Vegetable Research \\ and Development Center, P.O. Box 42, Shanhua, Tainan 741, Taiwan, R.O.C. \\ Accepted for publication 19 May 2005.
}

\begin{abstract}
Kim, K. S., Wang, T. C., and Yang, X. B. 2005. Simulation of apparent infection rate to predict severity of soybean rust using a fuzzy logic system. Phytopathology 95:1122-1131.

Few biologically based models to assess the risk of soybean rust have been developed because of difficulty in estimating variables related to infection rate of the disease. A fuzzy logic system, however, can estimate apparent infection rate by combining meteorological variables and biological criteria pertinent to soybean rust severity. In this study, a fuzzy

daily apparent infection rate of soybean rust and simulated disease severity based on population dynamics. In weekly simulation, the FLAIR model explained $>85 \%$ of variation in disease severity. In simulation of an entire epidemic period, the FLAIR model was able to predict disease severity accurately once initial values of disease severity were predicted accurately. Our results suggest that a model could be developed to determine apparent infection rate and an initial value of disease severity in advance using forecasted weather data, which would provide accurate prediction of severity of soybean rust before the start of a season.
\end{abstract} logic apparent infection rate (FLAIR) model was developed to simulate severity of soybean rust and validated using data from field experiments on two soybean cultivars, TK 5 and G 8587. The FLAIR model estimated
Additional keywords: logistic growth equation, risk assessment.
Mechanistic and empirical models have been developed to assess the risk of soybean rust, caused by Phakopsora pachyrhizi. Yang et al. (15) developed a model to predict severity of soybean rust analyzing data obtained from 73 sequential planting experiments in Taiwan. To predict disease severity, the model estimated infection rate, latent period, and senescence of uredia. Because this model required comprehensive information about the host, e.g., leaf area index, as well as the pathogen, it was challenging to apply it in practice. Using the same sequential planting experiment data as the training and validation sets, Batchelor et al. (1) applied neural networks to develop a prediction model for soybean rust severity. As input variables, the neural network model required degree-days to estimate soybean and $P$. pachyrhizi development, and determination of the first day on which disease was observed. The latter requirement, however, may hinder application of the model in fields since it would fail to release burden for regular field scouts.

Population dynamics can be used to predict soybean rust severity with a few parameters. For example, Van der Plank (12) suggested a logistic growth equation to predict disease severity using apparent infection rate as a key parameter. A mechanistic model, which is another approach to simulate disease severity, describes infection, sporulation, spore survival, inoculation, and incubation in mathematical terms. However, it requires precise and quantitative assessment of disease development, which is challenging to obtain such data under field conditions (6). Therefore, it would be preferable to apply population dynamics to predict disease severity when its parameters are determined.

To predict soybean rust severity using population dynamics, it is necessary to estimate its parameters, e.g., apparent infection

Corresponding author: K. S. Kim; E-mail address: luxkwang@yahoo.com

DOI: 10.1094/PHYTO-95-1122

(C) 2005 The American Phytopathological Society rate. A conventional regression approach can be used to estimate apparent infection rate. However, it would require quantitative values of measured apparent infection rate rather than quantitative ones. For example, a nonlinear regression approach, e.g., artificial neural networks, requires a training process to find the best-fit model by calculating mean square errors between measurements and predicted values (1). However, measured disease data may contain considerable errors even under well-designed and executed experiments (1). Furthermore, it is challenging to measure apparent infection rates for regression analysis that require a training set under different environmental conditions.

Fuzzy logic can be applied to develop a model without relying on precise and quantitative measurements $(2,6,9)$. For example, fuzzy logic has been used to represent uncertainties of input weather data in a generic pest risk model (8). Fuzzy logic is an extension of classic logic (17). While classic logic deals with exact logical reasoning using binary values, i.e., either "true" or "false", fuzzy logic handles vague, imprecise, or ambiguous representation of knowledge using a degree of truth, or partial membership in a set. In fuzzy logic, for example, a value of disease severity can be represented as "high" at the degree of 0,1 , or 0.4 ; the degree of 0 represents that disease severity is outside a "high" state, whereas the degree of 1 indicates that disease severity is completely in the "high" state. On the other hand, the value of 0.4 represents the degree to which a value of disease severity belongs to the "high" state.

Using fuzzy logic, it is possible to formulate decision rules based on available knowledge in order to estimate apparent infection rate. For example, a rule of fuzzy logic can be stated as follows: "if average temperature is low and host is healthy, then pathogen activity is low." Such rules have been applied to develop a model in different fields including epidemiology, ecology, and meteorology $(2,3,6,9)$. For instance, Orlandini et al. (6) used fuzzy logic to simulate grapevine powdery mildew development and infection in Italy. Kampichler et al. (2) simulated population of foliage-dwelling spiders in field margins using fuzzy logic. 
Kim et al. (3) also developed a leaf wetness duration (LWD) model based on a fuzzy logic system.

A fuzzy logic system, which is a collection of fuzzy rules, can be constructed using current knowledge to predict severity of soybean rust (4). For example, at dew period of $10 \mathrm{~h}$, infection of soybean rust was the greatest in the order of 22.5, 20, 25, 17.5, and $15^{\circ} \mathrm{C}$, whereas no infection was observed at $27.5^{\circ} \mathrm{C}(5)$. By incorporating such knowledge into a fuzzy logic system, it is possible to minimize dependency on precise and quantitative measurements to develop a model that describes the interaction between environment, soybean [Glycine max (L.) Merr.], and $P$. pachyrhizi. Applying population dynamics to the fuzzy logic system would also require simple calculation to predict disease severity compared with a mechanistic model. The objectives of this study were to develop a fuzzy logic system to estimate daily apparent infection rate of soybean rust using meteorological data and simulate soybean rust severity by applying the estimated apparent infection rate to a population dynamics model.

\section{THEORY AND APPROACHES}

Weather and disease severity data. Data obtained from sequential planting experiments of soybean at the Asian Vegetable Research and Development Center, Taiwan, were used to develop a daily apparent infection rate model. Determinate soybean cv. TK 5, which is susceptible, and indeterminate cv. G 8587, which is moderately resistant to Asian soybean rust (11), were planted on each date. TK 5 is less photoperiod sensitive and matures earlier than $\mathrm{G} 8587$. Each cultivar was planted in a plot ( 3 by $5 \mathrm{~m}^{2}$ ) separated by 2-m-wide corn (Zea mays L.) strips as described previously (16). Air temperature, dew period, and precipitation were monitored at 2-h intervals using an on-site weather station. A Taylor dew meter (10) was used to measure dew duration.

Experiments conducted in 1980 and 1981 were used for model development and validation, respectively. In 1980, a total of 46 weekly plantings were made from 7 January to 9 December. In the 1981 experiment, planting started on 21 December 1980 and continued through 21 September 1981, for a total of 27 planting dates at weekly intervals. In some cases, planting was delayed or hastened, which resulted in 158 disease epidemics in total. Beginning when rust was first observed in plots for each planting date, disease rating was recorded weekly by averaging measurements of four equal sections in each plot. Disease severity, which was the proportion of diseased leaf area to total leaf area, expressed as a percentage, was recorded eight times per planting date experiment. For cv. TK 5, the initial and final disease severity ranged from 0 to $28 \%$ and 21 to $98.5 \%$, respectively, and for cv. G 8587, 0 to $72 \%$ and 2.5 to $98.5 \%$, respectively, during 1980 and 1981 experiments. The field epidemics are described in the previous study (16).

Population dynamics equations. Increase in disease severity can be expressed as follows (12):

$$
\frac{d S_{t}}{d t}=r_{t} S_{t}\left(1-\frac{S_{t}}{100}\right)
$$

where $S_{t}=$ severity of soybean rust (\%) at a given date $t$ and $r_{t}=$ apparent infection rate. Daily apparent infection rate, $r_{t}$, is given by (12)

$$
r_{t}=\frac{R_{t-p} S_{t-p}}{S_{t}}
$$

where $R_{t}=$ basic infection rate at a given date $t$ and $p=$ latent period. $S_{t}$ can be calculated as follows:

$$
S_{t}=S_{t-1}+r_{t-1} S_{t-1}\left(1-\frac{S_{t-1}}{100}\right)
$$

where $t>0$. Under the assumption that $r_{t}$ is consistent throughout a season, solving equation 1 gives

$$
S_{t}=\frac{100 S_{0}}{S_{0}+\left(100-S_{0}\right) e^{-r t}}
$$

where $S_{0}=$ disease severity at the onset of outbreak and $r=$ apparent infection rate during the season.

Regression analysis. The nonlinear curve fitting tool of Sigma Plot (Systat Software Inc., Point Richmond, CA) was used to fit measured disease severity with equation 4 (Fig. 1). The co-

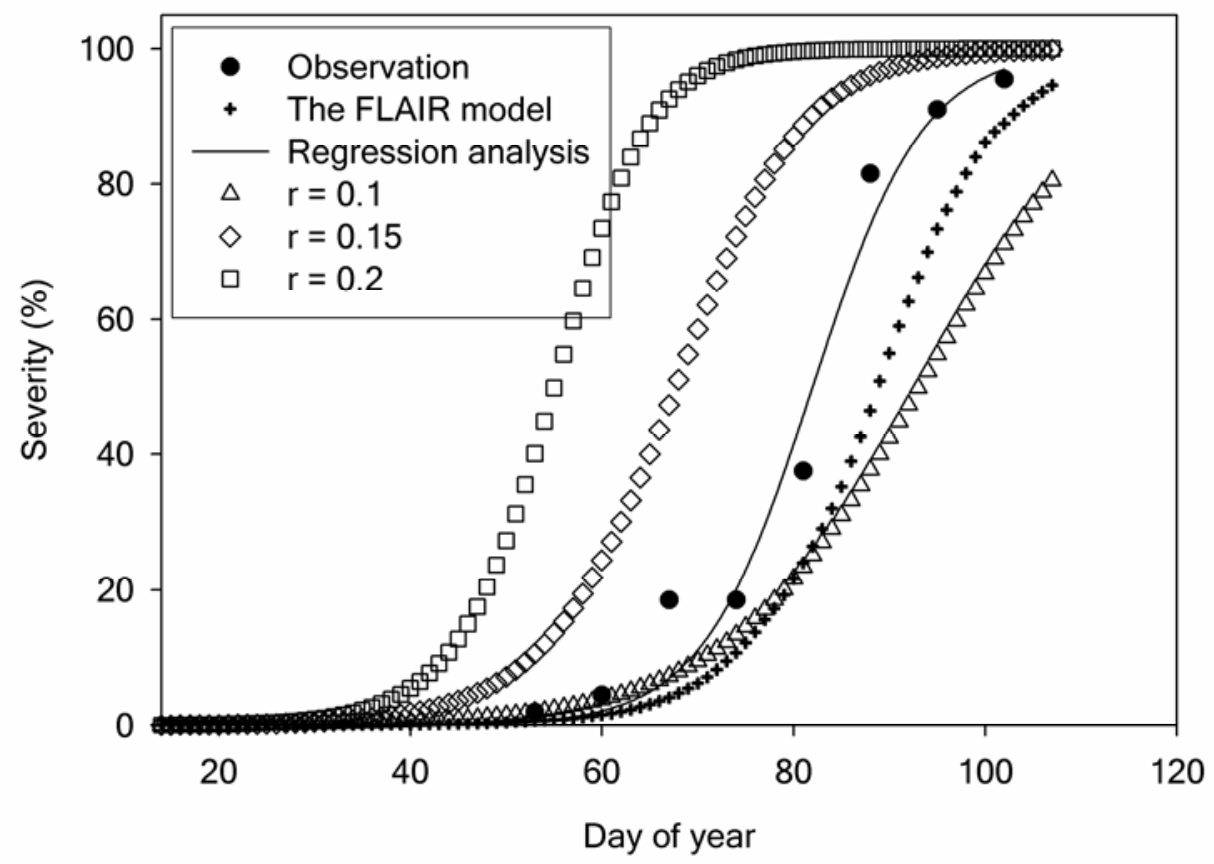

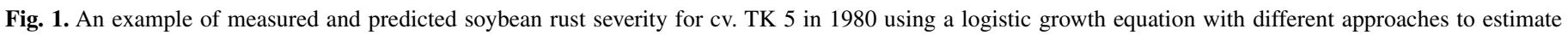

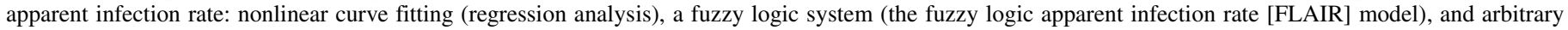

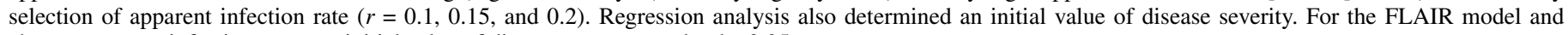
chosen apparent infection rates, an initial value of disease was assumed to be 0.05 . 
efficients of determination calculated from the regression analysis were used to assess goodness of fit between the logistic growth equation and measured severity of each epidemic. The date on which infection started to occur $(t=0)$ was set at 14 days after planting under the assumption that infection began as soon as soybean plants developed the first true leaves.

A fuzzy logic system to simulate severity of soybean rust. A fuzzy logic system, termed the fuzzy logic apparent infection rate (FLAIR) model, was developed to estimate a daily apparent infection rate (Appendix) and then apply it to equation 3 (Fig. 1). Daily maximum, minimum, and average air temperature during the night were used as input variables. To estimate daily apparent infection rate using a fuzzy logic system, rules and membership functions for these variables were defined (Appendix). Further description of fuzzy logic and fuzzy logic systems can be found in Kampichler et al. (2), Kim et al. (3), and Klir and Yuan (4).

At the beginning, decision rules and membership functions were defined from our experience. Using an iterative training set, they were later adjusted to yield the least error in simulating disease severity. Because disease severity was measured weekly, it was impossible to obtain measurements of daily apparent infection rate for a training process. Thus, root mean square error (RMSE) between measured and predicted values of disease severity from simulations during a 7-day period and an entire season was used to adjust the fuzzy logic system. The training set consisted of epidemics observed in 1980 for both cultivars. The validation set was prepared from 1981 epidemics for each cultivar. In this study, MATLAB (The Mathworks, Inc., Natick, MA) was used to develop the fuzzy logic system to estimate daily apparent infection rate.

Simulation of disease severity during a 7-day period. Using equation 3 , disease severity on a specific date could be determined when previously measured disease severity and estimated daily apparent infection rate were available. Starting from a date on which severity was measured, disease severity was simulated for a 7-day period using the FLAIR model that estimated daily apparent infection rate. Estimates of disease severity were compared with measurements to evaluate accuracy of the estimated daily apparent infection rate.

Sudden increase, decrease, or stagnation of disease severity was observed for some periods, which resulted in deviation from its logistic growth pattern. To identify such periods, the upper and lower limit of disease severity on a given date were calculated from measurements on a preceding date. Rearranging equation 4

TABLE 1. Distribution of estimated parameters obtained from regression analysis to fit a logistic growth equation to severity measurements of soybean rust caused by Phakopsora pachyrhizi during 1980 and 1981 in Taiwan

\begin{tabular}{lrrrrrr}
\hline & \multicolumn{1}{c}{$\mathrm{Q}^{\mathrm{a}}$} & $\mathrm{Q}^{\mathrm{a}}$ & Median & $\mathrm{Q}^{\mathrm{a}}$ & $\mathrm{Q}^{\mathrm{a}}$ & Mean \\
\hline $\mathrm{TK}^{\mathrm{b}}$ & $\left(N^{\mathrm{c}}=72\right)$ & & & & & \\
$r^{\mathrm{d}}$ & 0.004 & 0.136 & 0.173 & 0.237 & 0.454 & 0.190 \\
$\log _{10}\left(S_{0}\right)^{\mathrm{e}}$ & -12.396 & -3.103 & -1.885 & -0.544 & 1.498 & -2.241 \\
$R^{2}$ & 0.000 & 0.965 & 0.987 & 0.994 & 0.999 & 0.934 \\
$\mathrm{G} 8587^{\mathrm{b}}$ & $(N=86)$ & & & & & \\
$r$ & 0.008 & 0.047 & 0.081 & 0.136 & 0.774 & 0.105 \\
$\log _{10}\left(S_{0}\right)$ & -23.503 & -1.361 & -0.386 & 0.602 & 1.614 & -0.800 \\
$R^{2}$ & 0.000 & 0.827 & 0.942 & 0.983 & 1.000 & 0.819 \\
Overall & $(N=158)$ & & & & & \\
$r$ & 0.004 & 0.066 & 0.135 & 0.188 & 0.774 & 0.144 \\
$\log _{10}\left(S_{0}\right)$ & -23.503 & -2.356 & -0.794 & 0.383 & 1.614 & -1.456 \\
$R^{2}$ & 0.000 & 0.886 & 0.972 & 0.991 & 1.000 & 0.872 \\
\hline
\end{tabular}

${ }^{a}$ Q1, Q2, Q3, and Q4 indicate values of first, second, third, and fourth quartile of estimated parameters, respectively.

b TK 5 and G 8587 are susceptible and moderately resistant soybean cultivars to Asian soybean rust, respectively.

${ }^{c}$ The number of individual epidemics.

$\mathrm{d}$ Apparent infection rate.

${ }^{\mathrm{e}}$ Disease severity on the date 14 days after planting. for $S_{0}$ gives

$$
S_{0}=\frac{100 S_{t} e^{-r t}}{S_{t} e^{-r t}-S_{t}+100}
$$

When $S_{1}$ and $S_{2}$ are disease severity on $t_{1}$ and $t_{2}$, respectively, equation 5 can be written as

$$
\frac{100 S_{1} e^{-r t_{1}}}{S_{1} e^{-r t_{1}}-S_{1}+100}=\frac{100 S_{2} e^{-r t_{2}}}{S_{2} e^{-r t_{2}}-S_{2}+100}
$$

and rearranging equation 6 gives

$$
S_{2}=\frac{e^{\left(t_{2}-t_{1}\right) r} S_{1}}{1+\left(e^{\left(t_{2}-t_{1}\right) r}-1\right) \frac{S_{1}}{100}}
$$

where $t_{1}<t_{2}$. By assuming the upper and lower limit of $r$ to be 0.7 and 0.05 , respectively, the upper and lower limits of $S_{2}$ were determined using equation 7 . When measurements of disease severity at $t_{2}$ were between the upper and lower limits of $S_{2}$ for a 7-day period, the period was designated as a logistic growth period. Otherwise, it was considered to be a deviation period.

Simulation of disease severity during a season. When a date of initial infection and a value of $S_{0}$ were available, disease severity could be estimated for the remainder of a season using equation 3. Instead of using measurements of $S_{0}$, however, $S_{0}$ was estimated under the assumptions that onset of infection occurred 14 days after planting. For each epidemic, the value of $S_{0}$ was determined from regression analysis between equation 4 and measured disease severity. To simulate disease severity during a season using the FLAIR model, the value of $S_{0}$ was assumed to be consistent for all epidemics. Because cv. TK 5 is more susceptible to soybean rust than $\mathrm{cv}$. G 8587, $S_{0}$ values of 0.05 and 0.005 , respectively, were assumed for these cultivars. The $S_{0}$ value for each epidemic was later heuristically adjusted to minimize error in predicting disease severity. For heuristic adjustment, the value of $S_{0}$ increased or decreased arbitrary until values of $R^{2}$ between simulated and measured disease severity reached maximum for an epidemic. If several values of $S_{0}$ resulted in similar value of $R^{2}$, one that minimized error in predicting disease severity in an early stage ( $15 \%$ of disease severity) was preferred.

It would be preferable to estimate $S_{0}$ values preemptively rather than adjust them later. Instead of developing a model to estimate the $S_{0}$ values, however, a partition modeling analysis was performed to determine its sensitivity to variables that represent environmental and biological conditions, using JMP (SAS Institute Inc., Cary, NC). To provide surrogate environmental conditions, average temperature during the 30 -day period preceding planting was used. Planting date was also used as a surrogate variable to the initial inocula since experiment was conducted sequentially.

Apparent infection rate is another key parameter to simulate disease severity using a logistic growth equation. In this study, two kinds of apparent infection rate, daily and seasonal, were used to predict severity of soybean rust. Whereas daily apparent infection rate was estimated daily using the FLAIR model, seasonal apparent infection rate was consistent throughout a season. Considering that apparent infection rate of soybean rust ranged from 0.11 to 0.22 in Taiwan (11), seasonal apparent infection rate was selected, which was $0.1,0.15$, and 0.2 , and applied to equation 3 for each epidemic. Disease severity estimates for these seasonal apparent infection rates were later compared with severity estimates for the FLAIR model (Fig. 1). To determine seasonal apparent infection rate for the FLAIR model, the daily estimates of apparent infection rate were averaged during a period from 14 days after planting until the date on which soybean reached maturity. To classify seasons with respect to temperature conditions for soybean rust infection, air temperature was also averaged during the same period for each epidemic, which was designated as a seasonal average temperature. 
The index of agreement $(D)$, suggested by Willmott (13), was used to quantify accuracy in predicting disease severity for the FLAIR model and the selected seasonal apparent infection rates during each epidemic. $D$ was calculated as follows:

$$
D=1-\left[\sum_{i=1}^{N}\left(E_{i}-M_{i}\right)^{2} / \sum_{i=1}^{N}\left(\left|E_{i}^{\prime}\right|+\left|M_{i}^{\prime}\right|\right)^{2}\right], 0 \leq D \leq 1
$$

where $E_{i}$ and $M_{i}=$ an estimate and a measurement on a given date $i$, respectively, $E_{i}^{\prime}=E_{i}-\bar{M}, M_{i}^{\prime}=M_{i}-\bar{M}$, and $N=$ the number of measurements. A $D$ value of 1 indicates complete agreement between model estimates and measurements.

\section{RESULTS}

Regression analysis of measured disease severity. Fitting measured data to the logistic growth equation (equation 4) explained considerable variation in disease severity for most epi- demics (Table 1). Overall, about $75 \%$ of epidemics had $R^{2}$ values of $>0.89$. Values of $R^{2}$ were consistently high $(>0.95)$ at seasonal average temperatures between 20 and $25^{\circ} \mathrm{C}$, but dropped considerably outside this temperature range (Fig. 2A). Values of $R^{2}$ differed between cvs. TK 5 and G 8587 (Table 1). For example, $>75 \%$ of epidemics of TK 5 had $R^{2}$ values of $>0.97$, whereas $<50 \%$ of epidemics of $\mathrm{G} 8587$ had $R^{2}$ values of $>0.94$.

The distribution of seasonal apparent infection rate, $r$, obtained from regression analysis differed between TK 5 and G 8587 (Table 1). The $r$ values for TK 5 ranged from 0.004 to 0.454 , whereas those for G 8587 were between 0.008 and 0.774 . Nevertheless, the $r$ values for TK 5 generally exceeded those for $\mathrm{G}$ 8587. The $r$ values were related to seasonal average temperature (Fig. 2B).

In general, the initial values, $S_{0}$, obtained from regression analysis were smaller for TK 5 than for G 8587 (Table 1) and varied by planting date (Fig. 3A and B). It appeared that the $S_{0}$ values were related to average temperature during the 30-day

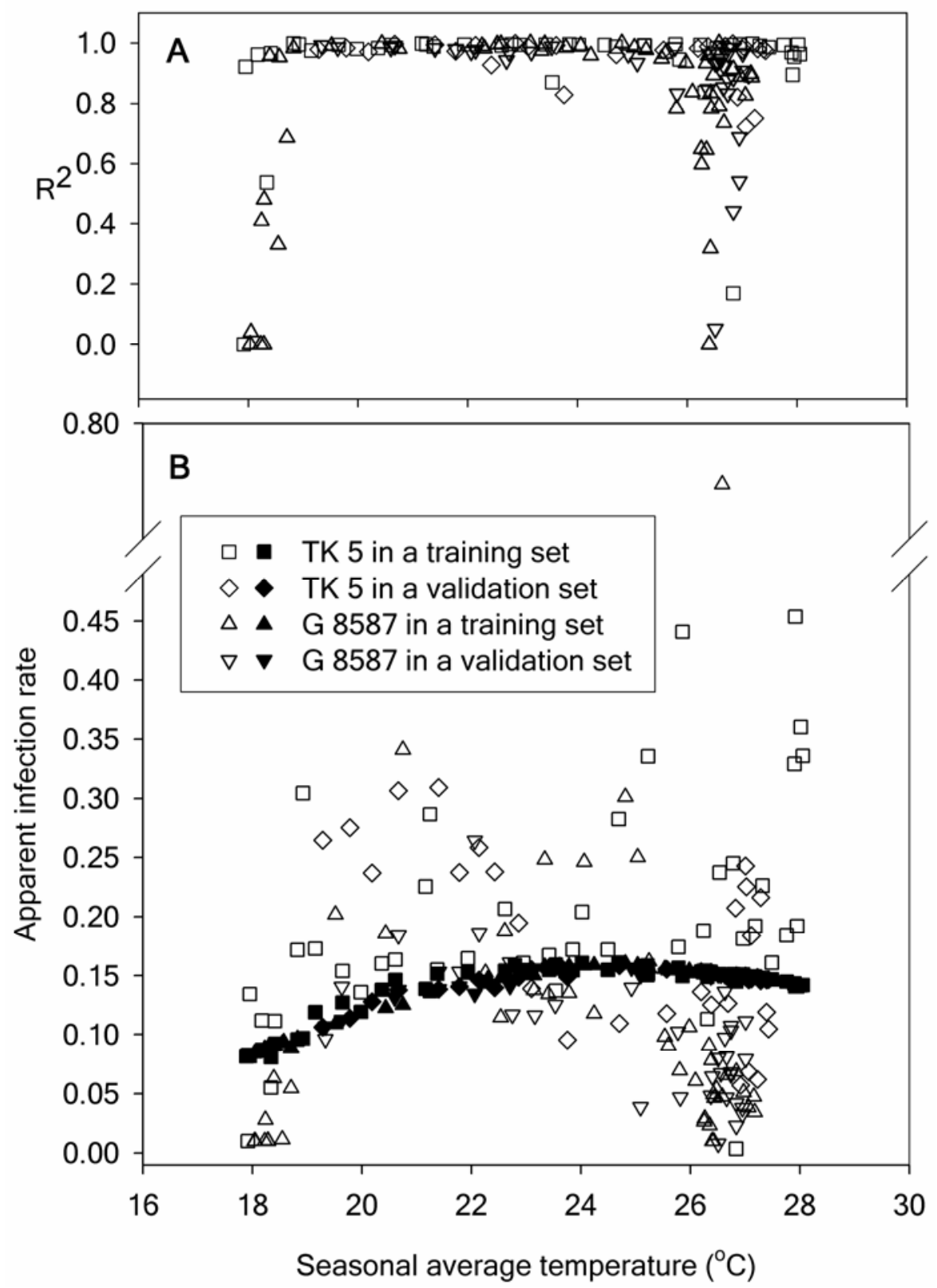

Fig. 2. The A, coefficient of determination and $\mathbf{B}$, seasonal apparent infection rate in relation to seasonal average temperature. Open and solid symbols indicate results from regression analysis and the fuzzy logic apparent infection rate (FLAIR) model, respectively. To obtain seasonal apparent infection rate estimates for FLAIR, its daily apparent infection rate estimates were averaged during a period from the date of 14 days after planting to the date on which soybean reached maturity. 
period preceding planting date. When a partition modeling analysis was performed, the coefficients of determination for TK 5 and G 8587 were 0.61 and 0.35 , respectively. Occasionally, values of $S_{0}$ dropped suddenly during summer and winter when average temperature before planting was $<18$ and $>24^{\circ} \mathrm{C}$, respectively, even though they tended to increase for the subsequent periods.

Estimation of seasonal apparent infection rate using the FLAIR model. The seasonal apparent infection rates for the FLAIR model ranged from 0.08 to 0.16 (Fig. 2B). They were related to the seasonal average temperature more closely than the apparent infection rates obtained from regression analysis. When they were fitted with a quadratic function of the season average temperature for both training and validation sets, the coefficient of determination was 0.95 and 0.99 for TK 5 and G 8587, respectively. The seasonal apparent infection rates were also similar for each cultivar.

Simulation of disease severity using the FLAIR model. In 7-day simulation, applying daily apparent infection rate estimates for the FLAIR model to the logistic growth equation explained $86.5 \%$ of variation of disease severity for both training and validation sets (Table 2). The FLAIR model yielded higher $R^{2}$ values for logistic growth periods than for other periods. The magnitude of RMSE for the FLAIR model was also smaller for logistic growth periods than for other periods.

In seasonal simulation, the $D$ values of predicted disease severity varied by seasonal average temperature (Table 3 ). For ex- ample, they were relatively low in most epidemics during which the average temperature was $<20^{\circ} \mathrm{C}$. When average temperature was $>25^{\circ} \mathrm{C}$, the FLAIR model simulated disease severity inaccurately in some cases. This pattern was consistent for both cvs. TK 5 and G 8587 (data not shown).

When the initial value, $S_{0}$, was assumed to be consistent for all epidemics, the average $D$ value for the FLAIR model was $<0.8$ (Table 3). Accuracy in predicting disease severity using the FLAIR model and seasonal apparent infection rate, $r$, differed by seasonal average temperature. When seasonal average temperature was $<20^{\circ} \mathrm{C}$, the $D$ values for the FLAIR model were similar to those for the $r$ value of 0.1 and greater than those for the $r$ value of 0.15 and 0.2 . During epidemics with seasonal average temperature $\geq 20^{\circ} \mathrm{C}$, the FLAIR model resulted in $D$ values similar to the $r$ value of 0.15 and 0.2 .

Most initial values of disease severity obtained from heuristic adjustment were between 1 and 0.001 , which varied less than values determined from regression analysis (Fig. 3C and D). Under similar temperature conditions preceding planting date, values for TK 5 tended to be greater than those for $\mathrm{G} 8587$. When a partition modeling analysis was performed, the coefficient of determination between the adjusted initial values, and the planting date and the average temperature before planting was 0.78 and 0.48 for TK 5 and G 8587, respectively.

After applying heuristically adjusted $S_{0}$ to equation 3, the FLAIR model substantially increased the $D$ values, which were 0.83 and
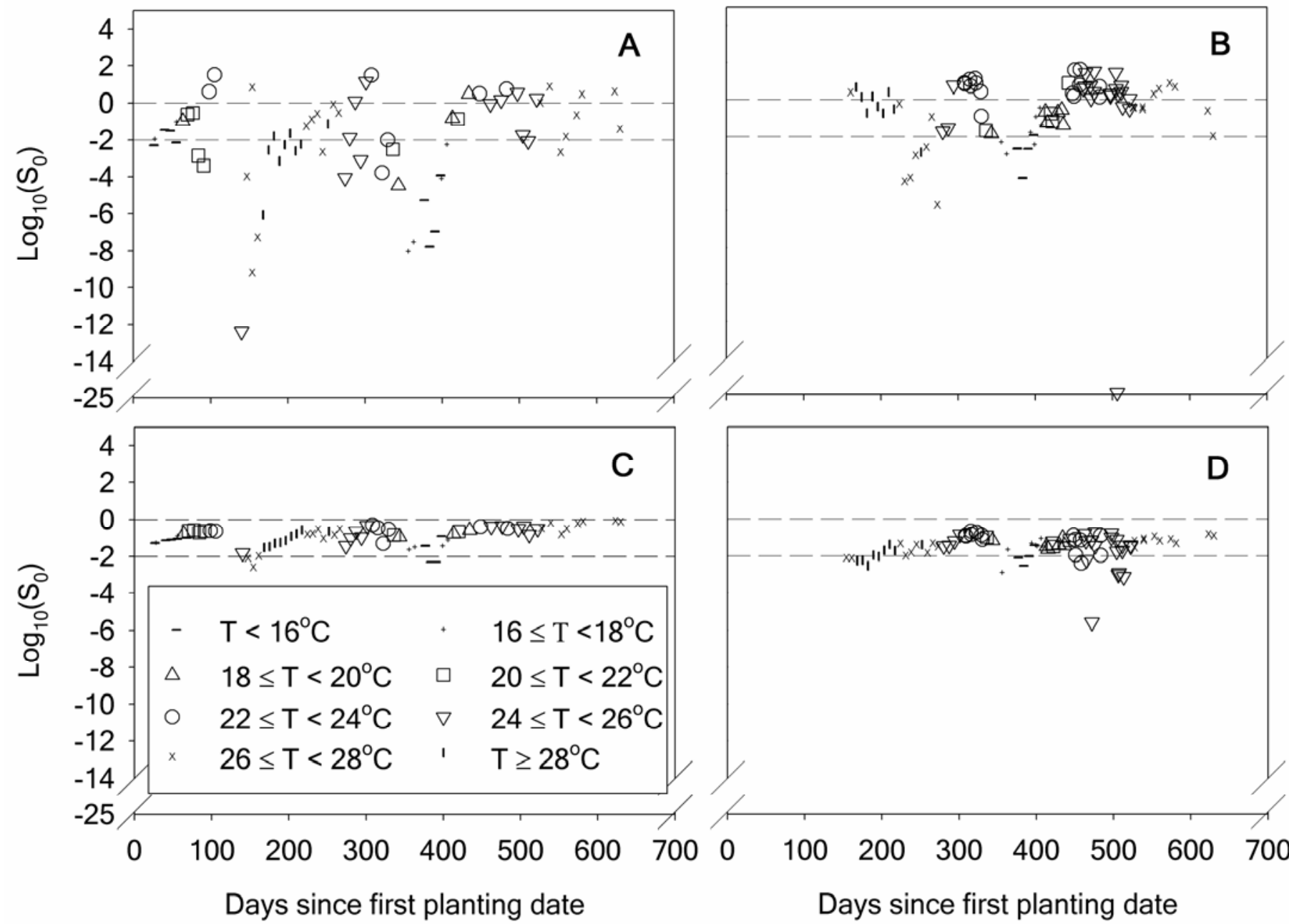

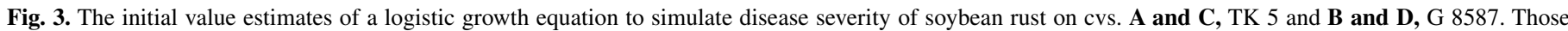

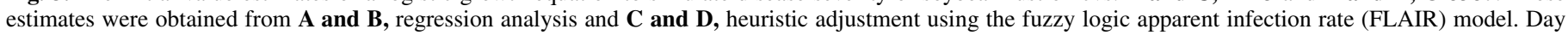

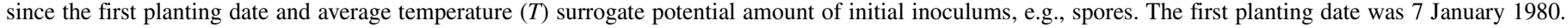

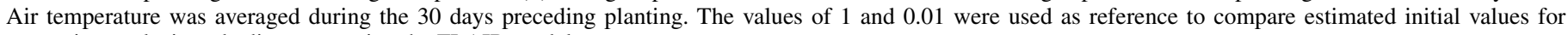
regression analysis and adjustment using the FLAIR model. 
0.9 on average for training and validation sets, respectively. The $D$ values for the FLAIR model and the $r$ value of 0.15 were similar to each other (Table 3 ). However, the $D$ values for the FLAIR model were greater than those for the $r$ value of 0.15 during seasons with average temperature $<20^{\circ} \mathrm{C}$.

\section{DISCUSSION}

This study demonstrates that severity of soybean rust can be simulated accurately by estimating daily apparent infection rate and applying it to a model of pathogen population dynamics. Using a fuzzy logic system, it was possible to develop a soybean rust severity model that estimates apparent infection rate with minimum dependency on its measurements. The FLAIR model also required simpler calculations than mechanistic models suggested by Orlandini et al. (6) and Yang et al. (15). However, the FLAIR model relied on an initial severity value that was determined heuristically, which substantially impairs the model's application in practice. Nevertheless, combining the FLAIR model with a model to predict the initial value of severity could provide

TABLE 2. Accuracy of soybean rust severity predicted for 7-day periods during 1980 and 1981 in Taiwan using a logistic growth equation of which daily apparent infection rate was estimated from the fuzzy logic apparent infection rate (FLAIR) model

\begin{tabular}{lccccccr}
\hline & \multicolumn{3}{c}{ All periods } & & \multicolumn{3}{c}{ Logistic growth periods $^{\mathrm{a}}$} \\
\cline { 2 - 3 } \cline { 6 - 8 } Cultivar & $N^{\mathrm{b}}$ & $R^{2 \mathrm{c}}$ & RMSE $^{\mathrm{d}}$ & & $N$ & $R^{2}$ & RMSE \\
\hline Training $^{\mathrm{e}}$ & & & & & & \\
TK 5 $^{\mathrm{f}}$ & 314 & 0.888 & 12.5 & & 220 & 0.907 & 11.5 \\
G 8587 & 357 & 0.871 & 13.6 & & 197 & 0.921 & 9.2 \\
All & 671 & 0.869 & 13.1 & & 417 & 0.911 & 10.5 \\
Validation & & & & & & \\
TK 5 & 183 & 0.865 & 12.9 & & 129 & 0.899 & 11.3 \\
G 8587 & 232 & 0.887 & 12.6 & & 147 & 0.938 & 8.3 \\
All & 415 & 0.868 & 12.7 & & 276 & 0.912 & 9.8 \\
\hline
\end{tabular}

a A 7-day period for which measured disease severity at the end of the period was between the upper and lower limits of disease severity determined using apparent infection rates of 0.7 and 0.05 , respectively.

$\mathrm{b}$ The number of 7-day periods.

c The coefficient of determination.

${ }^{\mathrm{d}}$ Root mean square error.

e Training and validation indicate a measured data set of disease severity in 1980 and 1981, respectively.

${ }^{f}$ TK 5 and G 8587 are susceptible and moderately resistant soybean cultivars to Asian soybean rust, respectively. a robust analysis tool to predict severity of soybean rust, determine fungicide spray timing, or select cultivars before a season.

To simulate pathogen populations and disease severity, several investigators have used a logistic growth equation whose key parameter was apparent infection rate $(11,12,16)$. In our work, use of population dynamics made it possible to simulate severity of soybean rust accurately since most epidemics had logistic growth pattern of disease severity (Table 1). However, such patterns differed by seasonal average temperature. For example, when a seasonal apparent infection rate was assumed to be $0.1,0.15$, and 0.2 , the corresponding $D$ values of estimated disease severity were dependent on seasonal average temperature. The $D$ values for the $r$ value of 0.1 were greater than those for the $r$ values of 0.15 and 0.2 when seasonal average temperature was $<20^{\circ} \mathrm{C}$. Otherwise, those for the $r$ value of 0.1 were substantially smaller than those for the $r$ values of 0.15 and 0.2 . In contrast, the $D$ values for the FLAIR model were not less than those for the $r$ values under different temperature conditions, suggesting that the FLAIR model would be effective to predict severity of soybean rust under field conditions (Table 3 ).

It is crucial to determine apparent infection rate in order to predict disease severity using a logistic growth equation. In practice, however, it is challenging to obtain measurements of apparent infection rate for model development and validation. Alternatively, regression analysis could be used to obtain estimates of apparent infection rate from measured disease severity. In our study, however, the seasonal apparent infection rates determined from regression analysis were often too large or too small. For example, they were frequently high $(>0.25)$ or low $(<0.10)$ under different temperature conditions (Table 1), whereas Tschanz and Wang (11) reported that apparent infection rate of soybean rust ranged from 0.11 to 0.22 in Taiwan. Therefore, it was questionable whether estimates of apparent infection rate determined from regression analysis could be used to develop a model that estimates apparent infection rate. In contrast, the FLAIR model estimated seasonal apparent infection rate between 0.08 and 0.16 without depending on measurements of apparent infection rate by applying knowledge of temperature response of $P$. pachyrhizi (Fig. 2B).

It seemed suitable to use the FLAIR model in order to estimate apparent infection rate for both TK 5 and G 8587 considering that the $R^{2}$ values and RMSE were similar between both cultivars in 7-day simulations (Table 2). Tschanz and Wang (11) reported that apparent infection rate for four cultivars including TK 5 and G 8587 was similar over a season, whereas a resistant cultivar,

TABLE 3. The mean of the index of agreement, suggested by Willmott (13), between measured and simulated disease severity of soybean rust using a logistic growth equation during 1980 and 1981 in Taiwan

\begin{tabular}{|c|c|c|c|c|c|c|c|c|c|}
\hline \multirow{2}{*}{$\begin{array}{l}\text { Average } \\
\text { temperature }^{\mathrm{a}}\end{array}$} & \multirow[b]{2}{*}{$N^{\mathrm{b}}$} & \multicolumn{4}{|c|}{ Assumed $S_{0}{ }^{c}$} & \multicolumn{4}{|c|}{ Adjusted $S_{0}{ }^{d}$} \\
\hline & & FLAIR $^{\mathrm{e}}$ & $r^{\mathrm{e}}=0.10$ & $r^{\mathrm{e}}=0.15$ & $r^{\mathrm{e}}=0.20$ & FLAIR & $r=0.10$ & $r=0.15$ & $r=0.20$ \\
\hline \multicolumn{10}{|l|}{ Training ${ }^{f}$} \\
\hline$<20^{\circ} \mathrm{C}$ & 24 & 0.56 & 0.56 & $0.46^{* * \mathrm{~g}}$ & $0.41 *$ & 0.66 & $0.49 * *$ & $0.42 * *$ & $0.41 * *$ \\
\hline $20-25^{\circ} \mathrm{C}$ & 28 & 0.83 & $0.52 * *$ & $0.80 *$ & $0.95 * *$ & 0.97 & $0.59 * *$ & $0.95 * *$ & $0.92 *$ \\
\hline$>25^{\circ} \mathrm{C}$ & 44 & 0.74 & $0.56^{* *}$ & $0.70 *$ & $0.67 *$ & 0.84 & $0.54 * *$ & 0.83 & $0.68 * *$ \\
\hline All & 96 & 0.72 & $0.55^{* *}$ & $0.67 * *$ & 0.69 & 0.83 & $0.54 * *$ & $0.76^{* *}$ & $0.68 * *$ \\
\hline \multicolumn{10}{|l|}{ Validation ${ }^{\mathrm{f}}$} \\
\hline$<20^{\circ} \mathrm{C}$ & 4 & 0.89 & 0.84 & 0.57 & $0.38 *$ & 0.92 & $0.87 *$ & $0.59 *$ & $0.38 * *$ \\
\hline $20-25$ & 22 & 0.80 & $0.59 * *$ & 0.77 & 0.81 & 0.95 & $0.64 * *$ & 0.95 & $0.83^{* *}$ \\
\hline$>25^{\circ} \mathrm{C}$ & 36 & 0.72 & $0.51 * *$ & 0.73 & $0.80 *$ & 0.86 & $0.69 * *$ & 0.86 & $0.73 * *$ \\
\hline All & 62 & 0.76 & $0.56 * *$ & 0.73 & 0.77 & 0.90 & $0.68 * *$ & $0.87 *$ & $0.75^{* *}$ \\
\hline
\end{tabular}

a Average temperature during a period from the date 14 days after planting to the date on which soybean reached maturity in an epidemic.

$\mathrm{b}$ The number of epidemics.

${ }^{\mathrm{c}}$ Initial value of disease severity was assumed to be 0.05 and 0.005 for TK 5 and G 8587, which are susceptible and moderately resistant soybean cultivars to Asian soybean rust, respectively.

${ }^{\mathrm{d}}$ Initial value of disease severity was later adjusted heuristically.

e Apparent infection rate estimated by the fuzzy logic apparent infection rate (FLAIR) model and selected arbitrarily, respectively.

${ }^{f}$ Training and validation sets indicate a measured data set of disease severity in Taiwan during 1980 and 1981, respectively.

$\mathrm{g} *$ and $* *$ indicate that the $D$ value was significantly different from that of the FLAIR model in paired $t$ test at $P<0.05$ and $P<0.01$, respectively. 
e.g., G 8587, had a much lower infection rate at an early stage of soybean rust infection. However, the coefficient of determination obtained from the partition modeling analysis was substantially different between TK 5 and G 8587. Therefore, it would be necessary to develop individual models to estimate initial value of severity for the former and the latter.

Once an initial value of disease severity was determined accurately, the FLAIR model seemed capable of predicting disease severity accurately since it predicted disease severity with high $D$ values (>0.8) after adjusting $S_{0}$ (Table 3 ). To forecast disease severity, it is necessary to predict initial epidemic conditions, e.g., viable spore density (14). After soybean rust is observed in a field, the FLAIR model may be able to simulate disease severity accurately using the measured initial value. However, it would be more practical to predict disease severity using accurate estimates of $S_{0}$. Our results showed that the initial values, $S_{0}$, were related to planting sequence and average temperature during the 30-day period before planting (Fig. 3). Identification of variables that represent biological and meteorological conditions influencing initial infection could improve the prediction of initial values of disease severity. This will require additional modeling efforts using various approaches, e.g., fuzzy logic systems, neural networks, classification and regression tree, genetic algorithm, or atmospheric transport models.

Previous soybean rust models did not incorporate biological and environmental processes in detail despite complex computation $(1,15)$. For example, Yang et al. (15) reported that it was impossible to integrate the effect of high temperature on survival or viability of uredia into their empirical model. A fuzzy logic system, in contrast, included relevant linguistic rules in order to simulate those processes. For example, rules such as, "if night average temperature is very low, then apparent infection rate is very low" and "if daily maximum temperature is high, then apparent infection rate is low," often resulted in a negative apparent infection rate, which corresponded to death or loss of viability of uredia.

Accuracy of the FLAIR model was related to goodness of fit to the logistic growth equation with measured disease severity. When seasonal average temperature was $>25^{\circ} \mathrm{C}$, many epidemics deviated from the logistic growth pattern (Fig. 2A). As a result, the FLAIR model was unsuccessful in simulating disease severity for those periods (Table 3). The FLAIR model also predicted disease severity with relatively low $D$ values during periods with average temperature $<20^{\circ} \mathrm{C}$ (Table 3 ). It is likely that biological responses of the pathogen under these conditions, e.g., diminished viability of uredia (7), caused such deviation from logistic growth. Therefore, the FLAIR model requires substantial improvement in simulating soybean rust severity during periods of limiting environmental conditions. The limited ability of the FLAIR model also suggests that another approach, e.g., a mechanistic model, may be needed to improve accuracy of simulation for such periods.

The FLAIR model estimates apparent infection rate without using dew periods or LWD as one of the input variables even though LWD is a determining factor for soybean rust infection (5). In general, it is difficult to obtain LWD data since standard weather stations rarely measure it. Models have been developed to estimate LWD (3), but most require at least hourly meteorological data including air temperature, relative humidity, and wind speed. To minimize requirement of input data and computation, a surrogate variable for LWD, average temperature during the night, was used. This approach assumes that moisture is not a limiting factor, which is valid in Taiwan (1). When hourly wetness data are available, however, night average temperature could be replaced with average temperature during wetness periods to predict disease severity using the FLAIR model.

Seasonal apparent infection rate for the FLAIR model was related to seasonal average temperature (Fig. 2B). Therefore, forecasts of air temperature could be used to predict apparent infection rate for a season in advance. Once seasonal apparent infection rate is determined using forecasted temperature, it may be possible to predict severity of soybean rust for an entire season. By predicting an initial value of severity, a logistic growth equation, e.g., equation 4, could be used to forecast severity of soybean rust even before the start of a season. This prospect provides scope for further modeling studies on apparent infection rate and initial value of severity.

\section{APPENDIX}

A fuzzy logic system is a collection of rules in which variables and their state are expressed in natural language, e.g., "temperature is high" or "temperature is low" (4). The linguistic terms representing the state of a variable were embodied using a fuzzy set. A fuzzy set is defined by a membership function $(\mu)$, which maps a numerical value of a variable to a partial degree of corresponding linguistic term. For example, when night average temperature is $16.6^{\circ} \mathrm{C}$, a membership function of the term "low" assigns 0.08 to the value. A geometric shape, e.g., a triangle, can be used to define a membership function (2). It is also possible for experts to create their own membership functions (4). Decision rules and membership functions to estimate daily apparent infection rate of soybean rust are represented in Table 4 and Figure 4.

Evaluation of a fuzzy logic system employs four fuzzy reasoning procedures: fuzzification, inference, aggregation, and defuzzification. First, a quantitative value of an input variable is converted to the linguistic term through fuzzification (Fig. 5A and B). As the inputs are fuzzified, inference evaluates all rules and determines strength of their truth in the form of a fuzzy set (Fig. 5C and D). Aggregation step combines all fuzzy sets obtained from inference procedure for each rule (Fig. 5E). Finally, defuzzification converts the combined fuzzy sets into a quantitative value, or a crisp value.

In fuzzification process, the degree of linguistic terms for an input variable is determined by a rule using the corresponding membership function. Since an input variable can be associated with multiple rules, the fuzzified value of an input value may vary

TABLE 4. Fuzzy rules to estimate daily apparent infection rate

\begin{tabular}{|c|c|c|}
\hline ID & Rules & Weight \\
\hline 1 & If night average temperature is very low, then daily apparent infection rate is very low & 1.00 \\
\hline 2 & If night average temperature is low, then daily apparent infection rate is moderate & 1.00 \\
\hline 3 & If night average temperature is moderate, then daily apparent infection rate is high & 0.90 \\
\hline 4 & If night average temperature is high, then daily apparent infection rate is extremely high & 0.70 \\
\hline 5 & If night average temperature is very high, then daily apparent infection rate is high & 0.60 \\
\hline 6 & If night average temperature is extremely high, then daily apparent infection rate is low & 0.45 \\
\hline 7 & If daily maximum temperature is low, then daily apparent infection rate is extremely high & 0.50 \\
\hline 8 & If daily maximum temperature is moderate, then daily apparent infection rate is high & 0.50 \\
\hline 9 & If daily maximum temperature is high, then daily apparent infection rate is low & 0.15 \\
\hline 10 & If daily minimum temperature is low, then daily apparent infection rate is very low & 0.50 \\
\hline 11 & If daily minimum temperature is moderate, then daily apparent infection rate is high & 1.00 \\
\hline 12 & If daily minimum temperature is high, then daily apparent infection rate is very high & 1.00 \\
\hline
\end{tabular}


according to those rules. For example, $15.6^{\circ} \mathrm{C}$ of daily minimum temperature is fuzzified as "moderate" at the degree of 0.24 for the following rule: "If daily minimum temperature is moderate, then apparent infection rate is high" (Fig. 5B; Table 4). In contrast, the same value of daily minimum temperature is fuzzified as "low" at the degree of 0 to evaluate the rule, "if daily minimum temperature is low, then apparent infection rate is very low."

Inference is a procedure to evaluate "if - then" statements of a fuzzy rule through a fuzzy operation and obtain a fuzzy set that represents its conclusion. A number of methods are available to define a fuzzy operation for an inference step. An implication operation, Imp, used in this study was given by

$$
\operatorname{Imp}\left(a, \mu_{C}(x)\right)=\min \left(a, \mu_{C}(x)\right)
$$

where $a$ is the degree of antecedent and $\mu_{C}(x)$ is membership function of consequence over $x \in[-\infty, \infty]$. For example, to determine fuzzy conclusion of the rule, "if daily minimum temperature is moderate, then apparent infection rate is high," the value of membership corresponding to the consequence term, "high" and 0.24 , which is a degree of antecedent from our previous example, are subjected to an implication operation (Fig. 5D).
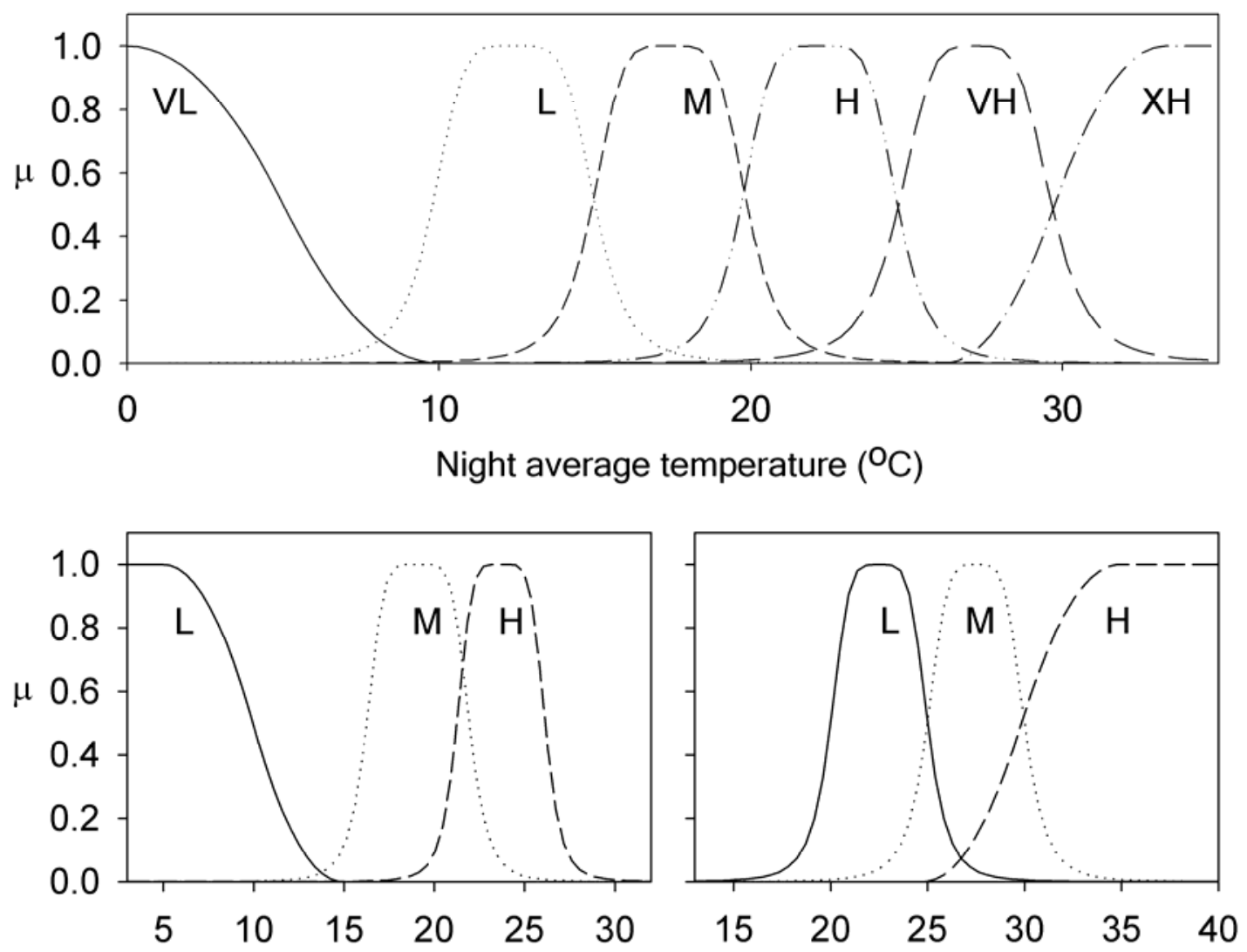

Daily minimum temperature $\left({ }^{\circ} \mathrm{C}\right)$ Daily maximum temperature $\left({ }^{\circ} \mathrm{C}\right)$

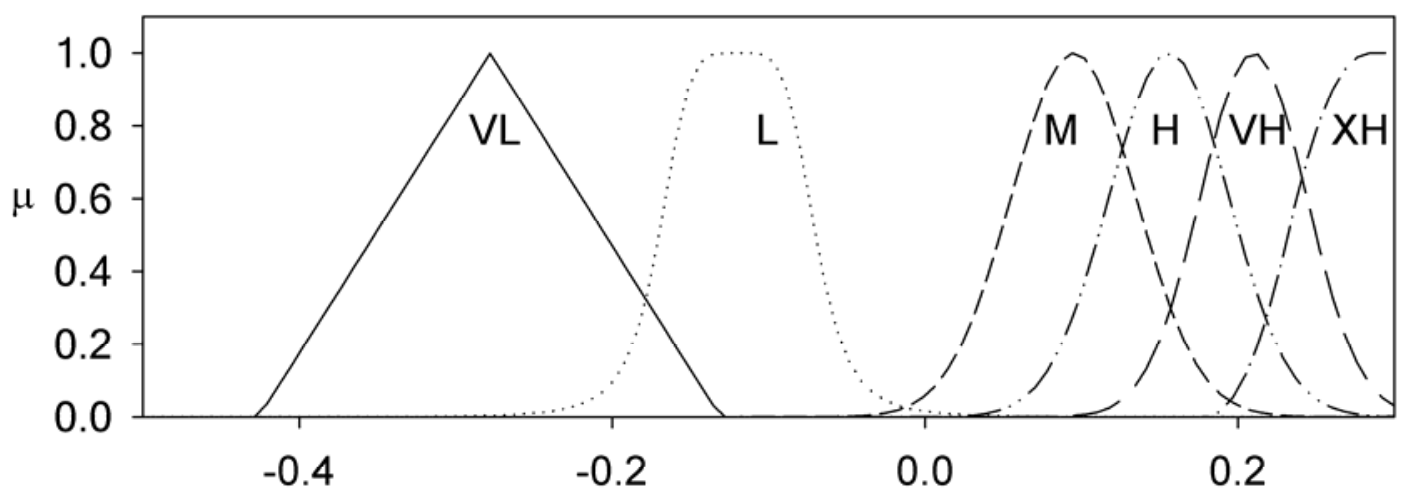

Apparent infection rate

Fig. 4. Membership functions $(\mu)$ for the variables of a fuzzy logic system to estimate daily apparent infection rate of soybean rust: $\mathbf{A}$, average temperature during night; $\mathbf{B}$, daily minimum temperature; $\mathbf{C}$, daily maximum temperature; and $\mathbf{D}$, daily apparent infection rate. L, M, H, V, and X indicate linguistic terms "low," "moderate," "high," "very," and "extremely," respectively. Using a membership function, a conventional numerical value is mapped to a value between 0 and 1 corresponding to a linguistic term. For example, membership functions for "low" and "moderate" of the variable, "night average temperature," convert the value of $16.6^{\circ} \mathrm{C}$ to "low" with the degree of 0.08 and "moderate" with the degree of 0.83 , respectively. 

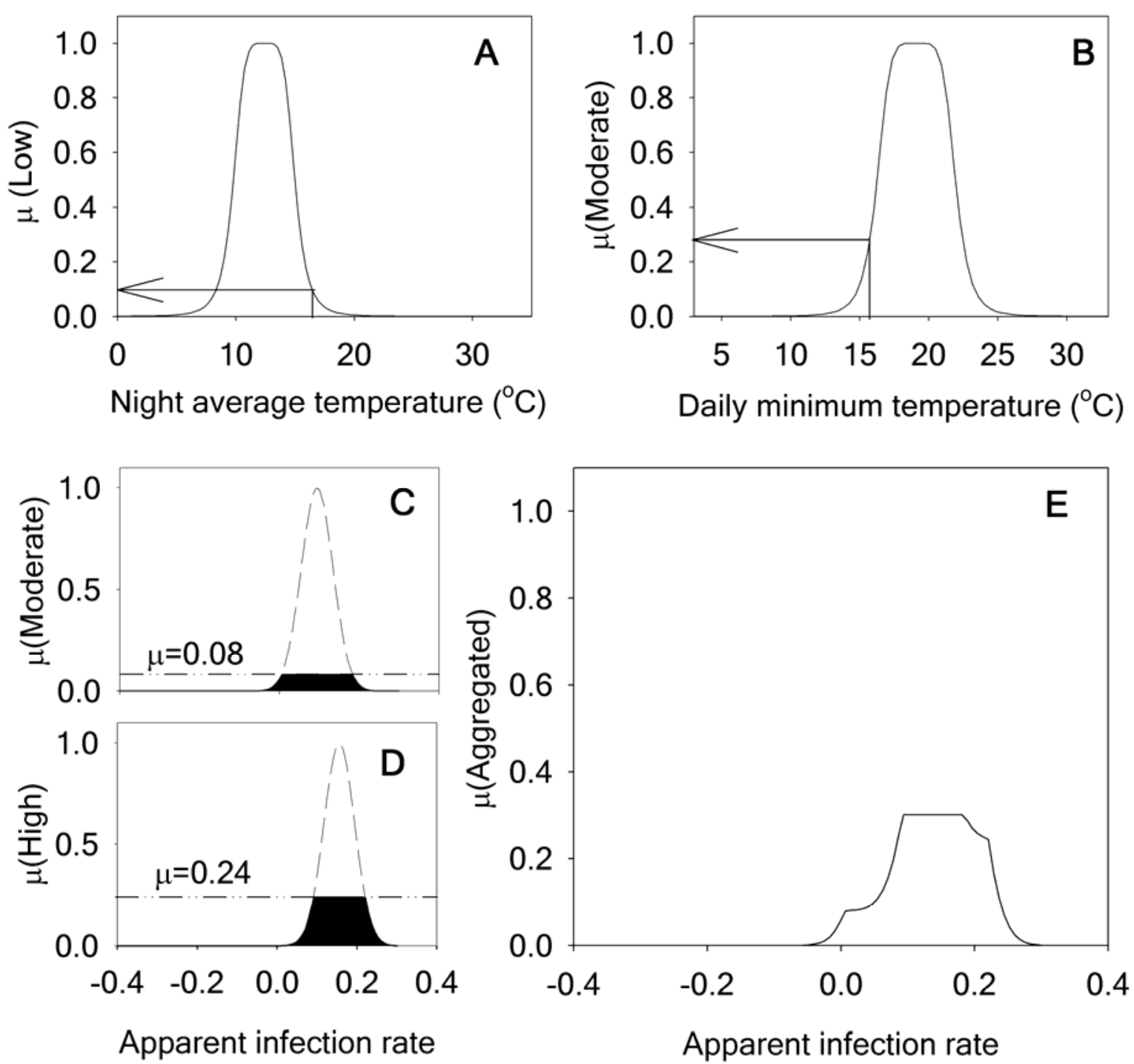

Fig. 5. An example of evaluation of a fuzzy logic system at $16.6^{\circ} \mathrm{C}$ night average temperature and $15.6^{\circ} \mathrm{C}$ daily minimum temperature to estimate daily apparent infection rate of soybean rust using rules, "if night average temperature is low, then apparent infection rate is moderate" and "if daily minimum temperature is moderate, then apparent infection rate is high": A and B, fuzzification step to assign a degree of linguistic term to the value of an input variable; C and D, inference step to derive a fuzzy set that represent conclusion of a rule after applying an implication operator; and $\mathbf{E}$, the result of an aggregation operation using fuzzy sets illustrated in $\mathbf{C}$ and $\mathbf{D} . \mu$ followed by a parenthesis indicates a membership function of a fuzzy set that represents a linguistic term of each variable within the parenthesis.

Now that the fuzzy logic system consists of multiple rules, multiple fuzzy sets that represent conclusion of each rule are obtained after the inference step. To combine these fuzzy sets, aggregation is performed using a fuzzy operation. In this study, an algebraic sum, probor, was used as follows:

$$
\operatorname{probor}\left(\mu_{i}(x), \mu_{j}(x)\right)=\mu_{i}(x)+\mu_{j}(x)-\mu_{i}(x) \mu_{j}(x)
$$

where $\mu_{i}(x)$ is the membership function of a fuzzy set that represents conclusion of $i$ th rule and $x \in[-\infty, \infty]$. For example, two fuzzy sets that represent conclusions of the rules, "if daily minimum temperature is moderate, then apparent infection rate is high" and "if night average temperature is low, then apparent infection rate is moderate," were combined into a single fuzzy set, as illustrated in Figure 5E.

The fuzzy set obtained from the aggregation procedure represents daily apparent infection rate simulated using the fuzzy logic system. However, defuzzification was necessary to translate the fuzzy set into a crisp value. In this study, the defuzzification operation was given by

$$
r=\frac{\int x \mu_{a g g}(x) d x}{\int \mu_{a g g}(x) d x}
$$

where $\mu_{\text {agg }}(x)$ is the membership function of a fuzzy set in the aggregation step and $x \in[-\infty, \infty]$.

\section{ACKNOWLEDGMENTS}

We thank A. T. Tschanz, USDA-APHIS, M. L. Gleason, Department of Plant Pathology, Iowa State University, and anonymous reviewers for helpful suggestions to improve the manuscript.

\section{LITERATURE CITED}

1. Batchelor, W. D., Yang, X. B., and Tschanz, A. T. 1997. Development of a neural network for soybean rust epidemics. Trans. ASAE 40:247-252.

2. Kampichler, C., Barthel, J., and Wieland, R. 2000. Species density of foliage-dwelling spiders in field margins: A simple, fuzzy rule-based model. Ecol. Model. 129:87-99. 
3. Kim, K. S., Taylor, S. E., and Gleason, M. L. 2004. Development and validation of a leaf wetness model using a fuzzy logic system. Agric. For. Meteorol. 127:53-64.

4. Klir, G. J., and Yuan, B. 1995. Fuzzy sets and fuzzy logic: Theory and applications. Prentice Hall PTR, Upper Saddle River, NJ.

5. Marchetti, M. A., Melching, J. S., and Bromfield, K. R. 1976. The effects of temperature and dew period on germination and infection by uredospores of Phakopsora pachyrhizi. Phytopathology 66:461-463.

6. Orlandini, S., Dalla Marta, A., D’Angelo, I., and Genesio, R. 2003. Application of fuzzy logic for the simulation of Plasmopara viticola using agrometeorological variables. OEPP/EPPO Bull. 33:415-420.

7. Patil, V. S., Wuike, R. V., Thakare, C. S., and Chirame, B. B. 1997. Viability of uredospores of Phakopsora pachyrhizi Syd. at different storage conditions. J. Maharashtra Agric. Univ. 22:260-261.

8. Scherm, H. 2000. Simulating uncertainty in climate-pest models with fuzzy numbers. Environ. Pollut. 108:373-379.

9. Stamou, G. P., Stamou, G. V., Papatheodorou, E. M., Argyropoulou, M. D., and Tzafestas, S. G. 2004. Population dynamics and life history tactics of arthropods from Mediterranean-type ecosystems. Oikos 104:98-108.
10. Taylor, C. F. 1956. A device for recording the duration of dew deposits. Plant Dis. Rep. 40:1025-1028.

11. Tschanz, A. T., and Wang, T. C. 1980. Soybean rust development and apparent infection rates at five locations in Taiwan. Prot. Ecol. 2:247-250.

12. Van der Plank, J. E. 1963. Plant Diseases: Epidemics and Control. Academic Press, New York.

13. Willmott, C. J. 1981. On the validation of models. Phys. Geogr. 2:184194.

14. Xu, X.-M., and Ridout, M. S. 1998. Effects of initial epidemic conditions, sporulation rate, and spore dispersal gradient on the spatio-temporal dynamics of plant disease epidemics. Phytopathology 88:1000-1012.

15. Yang, X. B., Dowler, W. M., and Tschanz, A. T. 1991. A simulation model for assessing soybean rust epidemics. J. Phytopathol. 133:187-200.

16. Yang, X. B., Royer, M. H., Tschanz, A. T., and Tsai, B. Y. 1990. Analysis and quantification of soybean rust epidemics from seventy-three sequential planting experiments. Phytopathology 80:1421-1427.

17. Zadeh, L. A. 1992. Knowledge representation in fuzzy logic. Pages 1-26 in: An Introduction to Fuzzy Logic Applications in Intelligent Systems. R. R. Yager and L. A. Zadeh, eds. Kluwer Academic, Norwell, MA. 